\title{
EVALUACIÓN DE LA RESILIENCIA EN MEXICANOS DIAGNOSTICADOS CON VIH: UN ESTUDIO COMPARATIVO
}

RESILIENCE ASSESSMENT IN MEXICANS DIAGNOSED WITH HIV: A COMPARATIVE STUDY

\section{JUAN CARLOS PLASCENCIA DE LA TORRE 1, CHRISTIAN LORENZO CASTELLANOS GUTIÉRREZ 2}

\author{
1., 2. UNIVERSIDAD DE GUADALAJARA, Guadalajara, México
}

\begin{abstract}
Objective: To assess the levels of resilience in Mexico. Diagnoses with HIV and compare the results of sociodemographic and clinical characteristics. Method: A quantitative, non-experimental, cross-sectional study with a descriptive and comparative scope, with 78 Mexicans diagnosed with HIV. The Mexican Resilience Scale (RESI-M) was used, it's formed by 43 items and six dimensions: Strength and Confidence in itself, Social Competence, Family Support, Social Support, and Personal Structure; the application was electronically through the Google Documents platform. Results: $67.9 \%$ of the evaluators showed high resilience, $28.2 \%$ moderate and only $3.8 \%$ low levels. There were no significant differences in any of the sociodemographic variables compared: $\operatorname{sex}(t=.657, p=.513)$, age range $(F=.553, p=.648)$, schooling $(F=.657, p=.082)$, and time with the disease ( $F=.657 ; p=.802$ ). Conclusions: People with HIV evaluated in this study have a high resilience capacity. Although there are no significant differences, men older than 35 years, with university education and the longer time with the positive diagnosis, are more resilient than women.
\end{abstract}

KEY WORDS: Resilience, HIV, Health Psychology.

\section{CÓMO CITAR / HOW TO CITE}

Plascencia De la torre, J. C. \& Castellanos Gutierrez, C. L. (2019). Evaluación de la resiliencia en mexicanos diagnosticados con $\mathrm{VIH}$ : Un estudio comparativo Efectos negativos del uso de benzodiacepinas en adultos mayores: una breve revisión. Salud \& Sociedad, 10(1), 52-64. doi: 10.22199/S07187475.2019.0001.00004 
El Virus de Inmunodeficiencia Humana (VIH) que causa el Síndrome de Inmunodeficiencia Adquirida (SIDA) es considerado en la actualidad como un problema de salud pública a nivel mundial, ya que genera un impacto negativo en la salud de las personas que lo portan y constituye un riesgo para la sociedad en general debido a su propagación constante, esto desde su aparición en la década de los 80's.

A partir del surgimiento del tratamiento antirretroviral a nivel mundial, el VIH-SIDA ha pasado de ser una enfermedad mortal a una enfermedad crónica degenerativa, por lo que las personas con un diagnostico positivo logran vivir cada vez más tiempo sin presentar síntomas de la enfermedad. El tratamiento antirretroviral permite reducir los niveles de carga viral en la sangre hasta un nivel indetectable, mejorando la calidad de vida, permitiendo llevar a cabo una vida cotidiana con actividades comunes y logrando así reducir las tasas de mortalidad en quienes lo padecen.

Aun así, partiendo de los avances de la medicina que busca mejorar las condiciones de vida de las personas con $\mathrm{VIH}$, existen factores de riesgo que influyen de manera directa en el progreso de la enfermedad y en el bienestar físico, psicológico y social del individuo tales como el estrés, la ansiedad, la depresión, el estigma de la sociedad, la situación económica de la persona, la violencia y la discriminación en contextos de bajo nivel educativo. Estos factores también impactan de manera negativa en la adherencia y la calidad de vida (Moskowitz, Hult, Bussolari, y Acree, 2009). No obstante, existen por otro lado, factores protectores como el apoyo social y la resiliencia, que permiten a las personas $\mathrm{VIH}+$ afrontar de manera positiva la enfermedad, dándoles la oportunidad de mejorar su condición y proyecto de vida.

\section{Conceptualización de Resiliencia}

El diccionario de la Real Academia Española (2017) define la resiliencia integrando dos concepciones, la primera relacionada con la física y las propiedades de los materiales, y la segunda que se acopla al objeto de estudio de este trabajo: la resiliencia es la capacidad que tienen los individuos para adaptarse ante un agente 0 estado perturbador. No obstante, algunos autores consideran que la resiliencia no sólo implica la superación del agente perturbador, sino la obtención de ganancias, resultados saludables o un mayor potencial después de afrontar dicha situación (Zautra, Hall y Murray, 2008; Forés, Grané, 2012; Noriega, Angulo y Angulo, 2015).

La resiliencia es un constructo abordado desde diversas disciplinas entre las que destacan la psicología, la antropología, la sociología, la filosofía, la economía y las ciencias de la salud (González, Valdez y Zavala, 2008). Desde la psicología y las ciencias de la salud, el afrontamiento de la adversidad con resultados positivos, en un primer momento se estudiaba bajo el término invulnerabilidad; no obstante, dicho vocablo implicaba una absoluta resistencia al daño y se entendía como una característica del individuo intrínseca e inmutable (Rutter, 1993). Se sustituyó invulnerabilidad por resiliencia debido a que este último término da cabida a la comprensión de la resistencia al daño como algo que no es absoluto, pues todo ser humano tiene sus límites, toma en cuenta que es una característica que reside tanto en el individuo como en el ambiente (González, Valdez y Zavala, 2008) y considera que los cambios del desarrollo influyen (Rutter, 1993; Noriega, Angulo y Angulo, 2015). Así pues, la resiliencia reconoce el sufrimiento y la lucha como aspectos que son parte del proceso (Noriega, Angulo y Angulo, 2015).

Desde la perspectiva de la psicología de la salud, el proceso de la resiliencia se da gracias a un cambio conjunto en la cognición, la afectividad y el comportamiento; la resiliencia implica tanto la resistencia al 
estrés que una persona puede soportar, como la sostenibilidad de los objetivos que dan sentido a la vida y que permiten seguir el curso satisfactorio de la misma, siendo esto último un aspecto crucial para la recuperación. (Zautra, Hall y Murray, 2008). Este proceso se forja al tener un encuentro con factores de riesgo, pero de manera que el individuo pueda hacer frente con éxito (Rutter, 1993; Noriega, Angulo y Angulo, 2015).

Resiliencia en enfermedades crónico degenerativas: El caso del VIH

Las enfermedades crónicas degenerativas son consideradas como una alteración orgánica funcional que genera cambios radicales en la vida de las personas; se caracterizan por ser persistentes en el tiempo, sin tener bien definido un inicio, curso y fin, puesto que algunas raramente logran tener una cura (Kralik, 2002; Stanton, Reverson y Tennen, 2007). Ante adversidades como lo es una enfermedad de esta índole, la resiliencia, considerada como la capacidad que tiene la persona de salir adelante después de una situación adversa y continuar con sus planes de vida (Zautra, Hall y Murray, 2008; Forés, Grané, 2012; Noriega, Angulo y Angulo, 2015) es un determinante protector de la salud, misma que favorece en la prevención de la enfermedad. La resiliencia como factor protector se ha estudiado en diversas enfermedades crónicas, tal es el caso del cáncer (Gotay, Isaacs y Pagano, 2004; Nelson Becker, 2006; Brix et al., 2008; Hou, Law, Yin y Fu 2010; Saravia, 2011), diabetes (Yi, et al., 2008; Mendéz, 2011; Denisco, 2011), reumatismo y dolor crónico (Bonanno, et al., 2002; Zautra, Jhonson y Davis, 2005; Strand, et al., 2006; Karoly y Ruehlamn, 2006; Smith y Zautra, 2008; Jhonson, Zautra y Going, 2008;), enfermedades coronarias (Chan, Lai y Woing, 2006), insuficiencia renal (Vinaccia y Quiceno, 2011) y sin dejar de lado el VIH-SIDA (Farber, Schwartz, Schaper y Moone, 2000; Carvalho, Morais, Koller y Piccinini, 2007; Orton, Griffiths, Green, y
Waterman, 2012; Bedón, 2013; Dale, et al., 2014).

Frente al VIH-SIDA, tema central de este estudio, un diagnóstico de seropositividad genera un impacto directo en el estado emocional de las personas, a lo que se requiere de la resiliencia y ciertas habilidades de afrontamiento para sobrellevar las consecuencias del diagnóstico. El proceso de resiliencia en pacientes con $\mathrm{VIH}$ es abordado por Carvalho (2007) como un proceso dinámico que permite a los pacientes superar la adversidad y obtener una mejor comprensión de su proceso de salud-enfermedad, así como los esquemas de tratamiento que se les proporciona. Algunas de las características resilientes que toda persona debe tener al enfrentar una enfermedad como lo es el VIH son confianza en sí mismo, apoyo familiar, fortaleza intrapsiquica, habilidad para la resolución de problemas y habilidad para las relaciones interpersonales. En ese mismo sentido, la literatura ha reportado que las características individuales de la persona, los recursos y competencias como la edad, capacidad cognitiva, sentido de propósito y creencias en cuanto al futuro son características esenciales que contribuyen a la resiliencia (Amzel et al., 2013).

La resiliencia estudiada en personas con $\mathrm{VIH}$ es uno de los temas poco estudiados, sin embargo, la investigación realizada a la fecha ha arrojado que aquellas personas que viven con VIH cuentan con altos niveles de resiliencia asociados a la adherencia al tratamiento y la calidad de vida (Farber, Scwartz, Schaper y Moone, 2000; Escobar, 2014; Espín, 2017), esto se puede deber a los avances que se han tenido en materia de salud, puesto que el VIH ya no es sinónimo de muerte y existe demasiada información al respecto lo que permite a las personas mantenerse actualizadas, afrontar de manera positiva un diagnóstico y llevar un estilo de vida normal. 
Por otro lado, en un estudio mexicano se buscó explorar las estrategias que utilizaban las personas para enfrentar el estigma asociado al VIH en el cual se encontró que dicha población logra reconstruir la representación de la enfermedad sin estigma como estrategia para enfrentar las situaciones de estigmatización por parte del contexto social, a la par en que mantienen oculto el diagnóstico y recurren a recursos sociales con el fin de mantenerse informados en cuanto a su condición de vida (Chong, Fernández, Huicochea, Álvarez y Leiva, 2012). En ese mismo sentido, Carvalho (2007) realizó un estudio con el objetivo de explicar la resiliencia y los aspectos clave de las personas que viven con VIH-SIDA, concluyendo que la resiliencia ayuda a disminuir la estigmatización y los prejuicios hacia la enfermedad y los pacientes; por otro lado permite cambiar las creencias en los mismos pacientes de que vivir con $\mathrm{VIH}$ es incompatible con el bienestar y la calidad de vida.

A pesar del reconocimiento de que las personas con VIH muestran una capacidad notable de recuperación ante la adversidad, aun se sabe poco sobre qué variables influyen en el proceso de la resiliencia. Ante lo ya mencionado, se establecieron como objetivos principales de esta investigación el evaluar los niveles de resiliencia en mexicanos diagnosticados con VIH. De igual forma se buscó comparar la resiliencia por sexo, rango de edad, escolaridad y antigüedad con el diagnóstico.

\section{MÉTODO}

\section{Diseño}

El presente estudio fue de corte cuantitativo, no experimental, de carácter transversal, con un alcance descriptivo y comparativo (Hernández, Fernández y Baptista, 2010)

\section{Población}

La población estuvo conformada por 78 personas de nacionalidad mexicana, mayores de edad, diagnosticados con $\mathrm{VIH}$, y que aceptaron participar de manera voluntaria en el estudio. Se empleó un muestreo no probabilístico por conveniencia, haciendo uso de la técnica bola de nieve y recurriendo al uso de las redes sociales para el contacto con los participantes.

\section{Instrumentos}

Para evaluar la resiliencia se utilizó la Escala de Resiliencia Mexicana (RESI-M) de Palomar y Gómez (2010), con un total de 43 items y con una confiabilidad de 0,967 ; dicha escala está conformada por las dimensiones de Fortaleza y Confianza en sí mismo, Competencia Social, Apoyo Familiar, Apoyo Social, y Estructura Personal. El instrumento tiene un formato tipo Likert. Se puntúa del 1 (Totalmente en desacuerdo) al 4 (Totalmente de acuerdo). La aplicación del instrumento fue en formato electrónico haciendo uso de las plataformas de Google Documents.

\section{Procedimientos}

Se contactó a los participantes a través de las redes sociales y correos electrónicos proporcionados, por el cual se les hizo llegar el instrumento en formato electrónico, con un consentimiento previo al llenado del instrumento donde se explicaron los objetivos del estudio y los tipos de resultados a obtener; todo se manejó de manera confidencial.

\section{Análisis Estadístico}

Se elaboró una base de datos haciendo uso del programa Excel y posteriormente del programa estadístico SPSS versión 20,0. Se determinaron frecuencias y porcentajes para las variables sociodemográficas; medias y desviación estándar para las variables numéricas. Se recurrió a la estadística diferencial para realizar comparaciones de la variable de estudio entre las variables sociodemográficas y clínicas de interés. 


\section{RESULTADOS}

De acuerdo al análisis descriptivo de las variables de estudio, la edad media de los participantes fue de 34.99 años (DE=9.590), de los cuales la mayoría se encontraban en un rango de edad entre los 18 y los 37 años. El $82.1 \%$ de los participantes fueron del sexo masculino, solteros y con educación media superior a superior. La mayoría de los participantes reportaron tener un tiempo de diagnóstico con la enfermedad menor a los cinco años y el $73 \%$ mencionó estar recibiendo tratamiento antirretroviral al momento del estudio (Tabla 1).

TABLA 1.

Características sociodemográficas de los participantes $(n=78)$.

\begin{tabular}{lccr}
\hline Características sociodemográficas & Frecuencia (n) & Porcentaje (\%) & Media (DE) \\
\hline Edad (años) & & & $34.99(9.590)$ \\
18-27 años & 17 & 21.8 & \\
$28-37$ años & 36 & 46.2 & \\
$38-47$ años & 12 & 15.4 & \\
>48 años & 13 & 17.7 & \\
Sexo & & & \\
Hombre & 64 & 82.1 \\
Mujer & 14 & 17.9 \\
Estado Civil & & & \\
Soltero & 61 & 78.2 \\
Casado/Unión Libre & 14 & 17.9 \\
$\quad$ Divorciado/Separado & 2 & 2.6 \\
$\quad$ Viudo & 1 & 1.3 \\
Escolaridad & & & \\
Educación Básica & 11 & 14.1 \\
Preparatoria & 14 & 17.9 \\
Carrera Técnica & 14 & 17.9 \\
Licenciatura & 27 & 34.6 \\
Posgrado & 12 & 15.4 \\
Antigüedad con el Diagnóstico & & \\
<1 año & 32 & 41 \\
1 a 5 años & 28 & 35.9 \\
5 a 10 años & 8 & 10.3 \\
>10 años & 10 & 12.8 \\
Recibe Tratamiento Antirretroviral & & \\
Sí & 73 & 93.6 \\
No & 5 & 6.4 \\
\hline Fuente: Elaboración propia & &
\end{tabular}

De manera global, los resultados de la Escala de Resiliencia Mexicana (RESI-M) detallan que los participantes portadores de VIH arrojaron una media de $\bar{x}=3.17$ (DE=0.543), misma que se ubica en un nivel de resiliencia alta. Al realizar una clasificación por niveles, se apreció que solo un $3.8 \%$ de los participantes obtuvieron bajos niveles de resiliencia, mientras que el resto se puntuó entre niveles moderados y altos (Tabla 2). En ese mismo sentido y teniendo en cuenta las dimensiones de la escala se apreció que los evaluados arrojaron mayores puntuaciones en las dimensiones de Apoyo social $(\bar{X}=3.38)$ y Fortaleza y confianza en sí mismo ( $\overline{\mathrm{X}}=3.22)$, en cuanto al resto de las dimensiones se obtuvieron los siguientes puntajes: Apoyo Familiar $(\overline{\mathrm{X}}=3.16)$, Competencia Social $(\overline{\mathrm{X}}=3.02)$ y Estructura personal $(\bar{X}=3.02)$. Todos considerados como niveles altos. 
TABLA 2.

Resultados generales de la resiliencia $(n=78)$.

\begin{tabular}{|c|c|c|c|c|c|c|}
\hline & $F$ & $\%$ & $\begin{array}{c}\text { Puntuación } \\
\text { Mínima }\end{array}$ & $\begin{array}{c}\text { Puntuación } \\
\text { Máxima }\end{array}$ & $\bar{x}$ & $\mathrm{DE}$ \\
\hline Baja Resiliencia & 3 & 3.8 & & & & \\
\hline Moderada Resiliencia & 22 & 28.2 & & & & \\
\hline Alta Resiliencia & 53 & 67.9 & & & & \\
\hline Resiliencia Total & & & 1.19 & 3.93 & 3.173 & 0.543 \\
\hline
\end{tabular}

Dentro de los objetivos del estudio, se planteó la comparación de los puntajes de resiliencia entre algunas de las variables sociodemográficas, tales como el sexo, rango de edad, escolaridad y tiempo con el diagnóstico de la enfermedad. En ese sentido se recurrió al uso de pruebas paramétricas, puesto que se cumplió con el criterio de normalidad aplicando la prueba de Kolmogorov donde se obtuvo un valor de $z=$ .898 y una significancia de .395 , lo que nos indica la normalidad de la distribución de la variable de estudio; así mismo el estadístico de Levene indicó la homogeneidad entre las varianzas de los grupos de comparación.
Una vez cumplidos los criterios para el uso de pruebas paramétricas, se obtuvieron las medias de la resiliencia total por sexo, donde los hombres obtuvieron un puntaje de $3.19(\mathrm{DE}=.565)$, mientras que en las mujeres fue de 3.08 ( $D E=.439$ ), donde se puede apreciar que los hombres puntúan mayor resiliencia que las mujeres, no obstante a través de la prueba $t$ de Student no se evidenciaron diferencias estadísticamente significativas $(t=.657 ; p=.513)$. En cuanto a las dimensiones de la escala, tampoco se encontraron diferencias significativas entre hombres y mujeres, sin embargo se aprecia que los hombres arrojan mayores puntajes que las mujeres en las dimensiones de Fortaleza y Confianza en sí mismo, Apoyo Familiar y Estructura personal (Tabla 3).

TABLA 3.

Medias y desviación estándar por sexo.

\begin{tabular}{|c|c|c|c|c|c|c|c|}
\hline \multirow{2}{*}{ Dimensiones de la Escala } & \multicolumn{2}{|c|}{ Hombres } & \multicolumn{2}{|c|}{ Mujeres } & \multirow{2}{*}{$\mathrm{t}$} & \multirow{2}{*}{$\mathrm{gl}$} & \multirow{2}{*}{$\mathrm{p}$} \\
\hline & Media & $\mathrm{DE}$ & Media & $\mathrm{DE}$ & & & \\
\hline Resiliencia Total & 3.19 & .565 & 3.08 & .439 & .657 & 76 & .513 \\
\hline Fortaleza y Confianza en sí mismo & 3.25 & .666 & 3.06 & .450 & .995 & 76 & .323 \\
\hline Competencia Social & 3.02 & .705 & 3.02 & .783 & .012 & 76 & .991 \\
\hline Apoyo Familiar & 3.17 & .816 & 3.11 & .830 & .240 & 76 & .811 \\
\hline Apoyo Social & 3.38 & .726 & 3.38 & .578 & -.006 & 76 & .995 \\
\hline Estructura Personal & 3.04 & .597 & 2.91 & .564 & .741 & 76 & .461 \\
\hline
\end{tabular}

Para llevar a cabo la comparación de la resiliencia tomando en cuenta el rango de edad, se utilizó un Análisis de la Varianza (ANOVA) en el cual no se encontraron diferencias significativas ( $F=.553 ; p=.648)$, sin embargo se evidencia que las personas mayores de 48 años tienden a ser más resilientes que los de menor edad (Tabla 4).
En ese mismo orden, se comparó la resiliencia tomando en cuenta el nivel de escolaridad en el cual no se encontraron diferencias estadísticamente significativas ( $F=.657 ; p=.082)$, sin embargo se puede apreciar que son las personas con estudios de posgrado los que arrojan mayores puntajes $\bar{X}=3.37(\mathrm{DE}=.278)$ en comparación 
con los que solo cuentan con estudios de educación básica quienes obtuvieron puntajes menores $\bar{X}=2.92(D E=.690)$ lo que indica que es esta población la menos resiliente ante el proceso de una enfermedad como el VIH (Tabla 5).

TABLA 4.

Resiliencia y rango de edad.

\begin{tabular}{lccccc}
\hline \multicolumn{1}{c}{ Rango de Edad } & $\overline{\mathrm{x}}$ & $\mathrm{DE}$ & $\mathrm{F}$ & $\mathrm{Gl}$ & $\mathrm{p}$ \\
\hline 18-27 años & 3.06 & .312 & & & \\
28-37 años & 3.15 & .581 & .553 & 77 & .648 \\
38-47 años & 3.22 & .548 & & & \\
$>$ 48 años & 3.31 & .682 & & & \\
\hline $\begin{array}{l}\text { Prueba ANOVA de un factor } \\
\text { Fuente: Elaboración propia }\end{array}$
\end{tabular}

TABLA 5.

Resiliencia y escolaridad.

\begin{tabular}{lccccc}
\hline \multicolumn{1}{c}{ Escolaridad } & $\overline{\mathrm{X}}$ & $\mathrm{DE}$ & $\mathrm{F}$ & $\mathrm{gl}$ & $\mathrm{p}$ \\
\hline Educación Básica & 2.92 & .690 & & & \\
Preparatoria & 3.33 & .382 & & & \\
Carrera Técnica & 2.93 & .626 & 2.164 & 77 & .082 \\
Licenciatura & 3.22 & .545 & & & \\
Posgrado & 3.37 & .278 & & & \\
\hline $\begin{array}{l}\text { Prueba ANOVA de un factor } \\
\text { Fuente: Elaboración propia }\end{array}$ & & & & & \\
\end{tabular}

Por último y de igual manera se utilizó una prueba ANOVA con el objetivo de comparar los puntajes de resiliencia teniendo en cuenta el tiempo con la enfermedad en donde no se encontraron diferencias significativas ( $F=.657 ; p=.802)$; sin embargo se evidencia que las personas con una antigüedad mayor a los 5 años son quienes se consideran más resilientes, en comparación con aquellos con un tiempo menor (Tabla 6).

TABLA 6.

Resiliencia y antigüedad con el diagnostico de VIH.

\begin{tabular}{lccccc}
\hline \multicolumn{1}{c}{ Antigüedad con el Dx } & $\overline{\mathrm{X}}$ & $\mathrm{DE}$ & $\mathrm{F}$ & $\mathrm{gl}$ & $\mathrm{p}$ \\
\hline$<1$ año & 3.12 & .536 & & & \\
1-5 años & 3.15 & .515 & .332 & 77 & .802 \\
$5-10$ años & 3.30 & .436 & & & \\
$>10$ años & 3.26 & .748 & & & \\
\hline Prueba ANOVA de un factor & & & & & \\
Fuente: Elaboración propia & & & & &
\end{tabular}

\section{DISCUSIÓN}

A partir de los resultados encontrados es posible afirmar, en primera instancia, que la muestra evaluada caracterizada por vivir con VIH cuenta con niveles moderados a altos de resiliencia, los cuales se consideran adecuados para afrontar el proceso de la enfermedad en sus vidas. Esta situación puede deberse a factores como la información que actualmente reciben por instituciones y profesionales de salud, misma que permiten terminar con los mitos y el estigma de vivir con VIH. Por otra parte, los avances en materia de farmacología han posibilitado contar con tratamiento altamente eficaz que permite en las personas con $\mathrm{VIH}$ disminuir su carga viral a niveles 
indetectables, contar con una óptima calidad de vida y seguir estilos de vida normal. Ante este hallazgo se han reportado resultados similares, tal es el caso de Escobar (2014) quien evaluó la resiliencia en personas con VIH encontrando que más del $75 \%$ de ellos contaban con altos niveles lo que permitía que fueran personas mayor adheridas a sus tratamientos. Por otro lado Mestanza y Tantaléan (2017) evaluaron a una muestra de 202 personas portadoras de VIH para identificar sus niveles de resiliencia y su relación con el autoconcepto, quienes concluyeron que las personas VIH cuentan con niveles adecuados de resiliencia y esta tiene una relación positiva con la variable de autoconcepto, por lo que un adecuado autoconcepto conlleva a altos niveles de resiliencia.

Al analizar los puntajes de las dimensiones conformadas en la escala, se aprecia que los evaluados obtuvieron mayores puntajes en Apoyo social por una parte, y por otra en la dimensión de Fortaleza y confianza en sí mismo. Esto permite comprender que el apoyo social que reciben las personas juega un papel importante en la resiliencia, puesto que las redes de apoyo dan un soporte al momento de afrontar las complejidades de la enfermedad, los efectos secundarios de los tratamientos y el estigma social. A su vez, el apoyo social genera fortaleza y confianza en sí mismo como parte del proceso de resiliencia lo que permite reconocer las capacidades de afrontamiento a cualquier situación adversa. En ese sentido, Skovdal y Daniel (2012) enfatizan que la agencia y la capacidad de las personas con $\mathrm{VIH}$ para dirigirse a través de las redes de apoyo es una vía clave hacia la resiliencia y debe fomentarse y reforzarse, en particular en los adolescentes $\mathrm{VIH}$ positivos. Ante este hallazgo, algo similar reportan Wollett, Cluver, Hatcher y Brahmbhatt (2016) quienes realizaron un estudio con personas $\mathrm{VIH+}$, quienes arrojaron altos niveles de resiliencia y de igual manera demostraron creencias pertinentes, que incluyen la creencia en el dominio y el reconocimiento de la fortaleza personal como consecuencia de la gestión de la adversidad por VIH. De igual forma se encontraron rasgos de carácter tales como la aceptación pragmática de la vida, la responsabilidad activa y una autoestima robusta. Las conductas sociales incluyeron la capacidad para acceder a los servicios médicos para satisfacer las necesidades de desarrollo, tener el deseo de apoyar y ayudar a los demás y desafiar el estigma relacionado con el VIH. Sin embargo es recomendable indagar más para identificar de donde proviene el apoyo social, si es directamente de la familia, amigos, trabajo u otras instituciones gubernamentales.

Al analizar si existían diferencias por sexo, se puede apreciar que no se encontraron diferencias estadísticamente significativas entre hombres y mujeres; sin embargo, se pudo observar que los hombres tienden a ser más resilientes. Tal condición posiblemente se deba a las asignaciones y expectativas sociales que implica el ser hombre en México, tales como la fortaleza, la resistencia y una supuesta invulnerabilidad. De igual forma, podría estar asociada la aún existente brecha entre sexos. [Tales resultados concuerdan con los hallazgos realizados por Saavedra y Villalta (2011), quienes además encontraron que no existe relación alguna entre la edad y los niveles de resiliencia. No obstante, otros estudios han encontrado diferencias significativas al realizar la comparación por sexos, es decir, los participantes hombres fueron más resilientes en comparación con las mujeres (González, 2010; Zavaleta, 2012).] No obstante, es recomendable profundizar más en esta variable para dilucidar si la capacidad resiliente que se encuentra en hombres es debida a aspectos culturales 0 a un factor sociodemográfico como lo es el nivel educativo, la edad, situación económica 0 lugar de residencia dentro del país.

Respecto al análisis de la resiliencia por rango de edad, no se encontraron diferencias significativas entre los grupos, sin embargo 
se aprecia que son las personas de mayor edad quienes reportan ser más resilientes que aquellos con edad más joven. Esto se podría deber al periodo de desarrollo donde la maduración física y psicológica atraviesa por diferentes periodos de la enfermedad, durante el cual no es igual la representación de la enfermedad en personas más jóvenes que en personas mayores; las personas de mayor edad cuentan con un nivel de madurez superior a los jóvenes, mayor experiencia de vida y mayores recursos de afrontamiento a las adversidades como lo es el VIH (Lorenzo, Alba, Rodriguez y Vargas, 2005). Esto brinda soporte a la idea de que los cambios del desarrollo influyen en el proceso de la resiliencia (Rutter, 1993; Noriega, Angulo y Angulo, 2015). No obstante, resulta pertinente revisar la relación existente entre edad y resiliencia con mayor puntualidad, con una muestra de mayor tamaño y con un estudio longitudinal, para poder realizar conclusiones más sólidas.

En cuanto al análisis por escolaridad, no se encontraron diferencias significativas entre los niveles educativos; no obstante se aprecia que son las personas con un nivel de posgrado las que reportan mayores puntajes de resiliencia comparado con aquellas con un nivel de educación básica. Partiendo de este hallazgo, que a pesar de no ser significativo se puede deducir que el nivel educativo juega un papel predominante en las personas que viven en una condición de $\mathrm{VIH}$, puesto que a mayor educación mayor serán las herramientas que permitirán hacer frente a situaciones adversas como lo es la enfermedad, así como fortalecer sus estados de salud a través de prácticas saludables. Estos resultados concuerdan con lo encontrado por Fernandes (2014), sin embargo difieren con lo encontrado por Dale, et al. (2014) quien concluye que son las personas con $\mathrm{VIH}$ de bajo nivel educativo son las que reportan mayor capacidad de adaptación y recuperación.

Por último, se realizaron análisis tomando en cuenta la antigüedad con la enfermedad en donde no se encontraron diferencias significativas, sin embargo se aprecia una mayor resiliencia en aquellas personas con una antigüedad con el diagnostico superior a los 5 años. Esta condición puede deberse al proceso de adaptación de la enfermedad, puesto que a mayor tiempo viviendo con el $\mathrm{VIH}$ las personas logran aceptar su condición de vida, acoplarla a sus proyectos y adaptarse a las exigencias que la enfermedad requiere como lo es la toma de antirretrovirales y asistir a citas médicas, generando mayor confianza y fortaleza hacia su persona. Algo parecido encontró Fernandes (2014) en su estudio donde evaluó a 159 personas con VIH encontrando que las personas con una antigüedad de 23 a 28 años de diagnóstico arrojaban mayores niveles de resiliencia en comparación con aquellos con una antigüedad con el diagnostico de 13 a 17 años.

\section{CONCLUSIONES}

A manera de conclusión, las personas que actualmente viven en una condición de $\mathrm{VIH}$ cuentan con una alta capacidad de afrontar las situaciones adversas que se les presentan como lo es la misma enfermedad. A pesar de no haber encontrado diferencias estadísticamente significativas, se pudo observar una tendencia en los hombres, con una edad por encima de los 35 años, con escolaridad universitaria y mayor tiempo con el diagnóstico positivo a ser más resilientes, en comparación con las mujeres. Sin embargo, este hallazgo puede deberse a diferencias socioculturales de la población de estudio 0 al tamaño muestral. Aun así este estudio contribuye a determinar la capacidad de afrontamiento del VIH en personas con un estado serológico positivo y los recursos que los llevan a considerarse resilientes.

Durante la elaboración de este estudio se encontró como limitante el tamaño de muestra, puesto que es incidental de personas en esta condición, por lo que los resultados no pueden generalizarse a toda la población. Es recomendable realizar estudios similares con una muestra más 
amplia, aleatoria y homogénea. También se recomienda profundizar en el tema de la resiliencia desde una perspectiva de investigación tanto cuantitativa como cualitativa con el fin de comprender los factores que contribuyen al fortalecimiento de la misma en personas con $\mathrm{VIH}$.

Por otro lado es de suma importancia la participación de los profesionales de la salud, especialmente de la Psicología y sus distintos campos de aplicación, ya no dirigidos solamente a cubrir las necesidades de quienes viven con $\mathrm{VIH}$, sino también a fortalecer las capacidades de afrontamiento en el proceso de la enfermedad. Se aprecia que quienes viven bajo esta condición cuentan con niveles adecuados de resiliencia, por lo que se plantea la necesidad de trabajar en conjunto con el sector salud, bajo un modelo de promoción que busque fortalecer y mantener los recursos que los individuos tienen en sí mismo y en su contexto, recursos tales como la confianza, la búsqueda de redes de apoyo social y familiar, y el desarrollo de un plan de vida integral. Esto permitirá en ellos incrementar sus niveles de adherencia terapéutica, desarrollar estilos de vida saludable y optimizar su calidad de vida.

\section{REFERENCIAS}

Amzel, A., Toska, E., Lovich, R.,Widyono,M., Patel, T., Foti, C., \& Altschuler, J. (2013). Promoting a combination approach to paediatric HIV psychosocial support. AIDS, 27, 147-157.

Bedón López, Y. (2013). Resilencia y adherencia al TARGA en pacientes con $\mathrm{VIH} /$ Sida del Hospital San Juan Bautista de Huaral. Revista PsiqueMag, 2(1), 5163. Recuperado de http://www.ucvlima.edu.pe/psiquemag/i ndex.htm

Block, R. G. (2009). Is it just me? experiences of HIV-related stigma. Journal of HIVIAIDS \& Social Services, 8, 1-19.

Bonanno, G. A., Ho, S. M., Chan, J. C., Kwong, R. S., Cheung, C. K., Wong,
C.P., \& Wong, V. C. (2008). Psychological resilience and dysfunction among hospitalized survivors of the SARS epidemic in Hong Kong: a latent class approach. Health Psychology, 27(5), 659-667.

Bonanno, G. A., Wortman, C. B., Lehman, D. R., Tweed, R. G., Haring, M., Sonnega, J., Carr, D., \& Nesse, R.M. (2002). Resilience to loss and chronic grief: $A$ prospective study from pre loss to 18months post loss. Journal of Personality and Social Psychology, 83, 1150-1164.

Brix, C., Schleußner, C., Füller, J., Röhrig, B., Wendt, T.G. \& Strauß, B. (2008). The need for psychosocial support and its determinants in a sample of patients undergoing radiooncological treatment of cáncer. Journal of Psychosomatic Research, 65(6), 541-548

Carvalho, F. (2007). Los factores protección $y$ resiliencia en las personas que viven con $\mathrm{VIH}$-SIDA. (Tesis de licenciatura inédita). Universidad Federal do Rio Grande do Sul, Porto Alegre, Brasil.

Carvalho, F. T., Morais, N. A., Koller, S. H. \& Piccinini, C. A. (2007). Fatores de proteção relacionados à promoção de resiliência em pessoas que vivem com HIVIAIDS. Cadernos de Saúde Pública, 23, 2023-2033.

Chan, I. W. S., Lai, J. C. L. \& Wong, K. W. N. (2006). Resilience is associated with better recovery in Chinese people diagnosed with coronary heart disease. Psychology \& Health, 21, 335-349.

Chong, F., Fernández, C., Huicochea L., Álvarez, G. y Leiva, R., (2012). Estrategias de personas con VIH para enfrentar el estigma asociado al VIH/SIDA. Pacientes del hospital General de Huixtla, Chiapas. Estudios Fronterizos, Nueva Época 13(25). Recuperado de: http://www.scielo.org.mx/pdf/estfro/v13 n25/v13n25a2.pdf

Dale, S. K., Cohen, M. H., Kelso, G. A., Cruise, R. C., Weber, K. M., Watson, C., Burke, J.K., \& Brody, L.R. (2014). Resilience among women with HIV: 
impact of silencing the self and socioeconomic factors. Sex Roles, 70, 221-231.

Denisco, S. (2011). Exploring the relationship between resilience and diabetes outcomes in African Americans. Journal American Academy of Nurse Practitioners, 23, 602- 610.

Escobar Jijón, J. (2014). La resiliencia y su relación con la adherencia al tratamiento en usuarios con $\mathrm{VIH}$ de la Unidad de Atención Integral PVV del Hospital Enrique Garcés. [Tesis de Pregrado Inédita]. Universidad Central del Ecuador.

Espín Hernadez, K.A. (2017). Pacientes con diagnóstico de VIH positivo y su capacidad de resiliencia ante los niveles de ansiedad y depresión provocados por la enfermedad en el Hospital San Vicente de Paul Periodo 2016-2017. [Tesis Inédita]. Universidad Técnica del Norte.

Farber, E. W., Schwartz, J. A. J., Schaper, P. E., \& Moone, D. J. (2000). Resilience factors associated with adaptation to HIV disease. Psychosomatics, 41, 140146.

Fernandes de Araujo, L. (2014). Autoeficacia, Conocimiento, Resiliencia y Preocupación por el VIH-SIDA: Diferencias entre infectados y no infectados por el VIH. [Tesis Doctoral]. Universidad de Granada, España.

Forés, A., Grané, J. (2012). La resiliencia en entornos socioeducativos. Madrid, España: Narcea. Recuperado de: https://books.google.com.mx/books?id= rpIPIxouciAC\&printsec=frontcover\&hl=e s\&source=gbs_ge_summary_r\&cad $=0 \#$ $\mathrm{v}=$ onepage $\& q \& \mathrm{f}=$ false

González N. (2008). Factores de la resiliencia presentes en adolescentes mexicanos. [Tesis para optar el grado de bachiller en Obstetricia.] México.

González, N., Valdez, J. y Zavala, Y. (2008). Resiliencia en adolescentes mexicanos. Enseñanza e Investigación en Psicología, 13(1), pp.41-52. Recuperado de: http://www.redalyc.org/articulo.oa?id=2 9213104

Gotay, C.C., Isaacs, P., \& Pagano, I. (2004). Quality of life in patients who survive a dire prognosis compared to control cancer survivors. Psycho- Oncology, 13, 882-892.

Hernández, R., Fernández, C. y Baptista, P. (2014). Metodología de la investigación. México: McGraw Hill.

Hou, W. K., Law, C. C., Yin, J. \& Fu, Y. T. (2010). Resource loss, resource gain, and psychological resilience and dysfunction following cancer diagnosis: a growth mixture modeling approach. Health Psychology, 29, 484-495.

Johnson, L., Zautra, A. J., \& Going, S. (2008). Adaptation to Early Knee Osteoarthritis: The role of risk, resilience, and disease severity on pain and physical functioning. Annals of Behavioral Medicine, 36(1), 70-80.

Karoly, P., \& Ruehlman, L. S. (2006). Psychological "resilience" and its correlates in chronic pain: findings from a national community sample. Pain, 123(1-2), 90-97.

Kralik, D. (2002). The quest for ordinariness: transition experienced by midlife women living with chronic illness. Journal of Advanced Nursing, 39(2), 146-154.

Lorenzo Ruiz, A.; Alba Pérez, L.; Rodriguez Reyes, M., Vargas López, R. (2005). Psicología de la Salud en la atención a niños y adolescentes. En Hernández y Grau. Psicología de la Salud. Fundamentos y Aplicaciones. Universidad de Guadalajara.

Méndez, G. (2011). Resiliencia en pacientes diabéticos (estudio realizado en el patronato de diabéticos de Quetzaltenango), [Tesis de licenciatura Inédita]. Universidad Rafael Landívar, Guatemala.

Mestanza Sánchez, R., Tantaleán Chávez, M.S. (2017). Capacidad de Resiliencia y Autoconcepto en Pacientes con VIHSIDA de la Estrategia Sanitaria de ITSVIH-SIDA y Hepatitis B, Hospital Amazonico Yarinacocha-Pucallpa. 
[Tesis inédita] Universidad Nacional de San Martin Tarapoto. Perú.

Moskowitz, J.T., Hult, J.R., Bussolari, C., \& Acree, M. (2009). What works in coping with HIV? A meta-analysis with implications for coping with serious illness. Psychological Bulletin, 135(1), 121.

Nelson-Becker, H. B. (2006). Voices of resilience: older adults in hospice care. Journal of social work in end-of-life $y$ palliative care, 2(3), 87- 106

Noriega, G., Angulo, B. y Angulo, G. (2015). La resiliencia en la educación, la escuela y la vida. Perspectivas docentes, 58, 42-48. Recuperado de: https://dialnet.unirioja.es/ejemplar/4831 64

Orton, L., Griffiths, J., Green, M. \& Waterman, H. (2012). Resilience among asylum seekers living with HIV. BMC Public Health, k, 926-936.

Palomar Lever, J.; Gómez Valdez, N. (2010). Desarrollo de una escala de medición de la resiliencia con mexicanos (RESIM). Interdisciplinaria, 27(1), 7-22.

Real Academia Española (2017). Diccionario de la lengua española. Recuperado de: http://dle.rae.es/?id=WA5onlw

Rutter, M. (1993). Resilience: some conceptual considerations. Journal of Adolescent Healt, 14(8), 626-631. doi: 10.1016/1054-139X(93)90196-V

Saavedra E, Villalta M. (2011). Comparar los puntajes generales y por factores de la variable Resiliencia, en sujetos de diferentes tramos de edad y de ambos sexos, a través de la prueba SV-RES, durante el segundo semestre de 2011. [Tesis para optar la licenciatura en Enfermería]. Universidad Nacional de Trujillo. Perú.

Saravia, J. (2011). Características de la resiliencia de los pacientes con cáncer terminal que se encuentran en aislamiento en el área de adultos del Hospital General San Juan de Dios. [Tesis de licenciatura Inédita]. Universidad Rafael Landívar, Guatemala.
Skovdal, M. \& Daniel, M. (2012). Resilience through participation and copingenabling social environments: The case of HIV-affected children in sub-Saharan Africa. African Journal of AIDS Research, 11(3), 153-164.

Smith, B. W. \& Zautra, A. J. (2008). Vulnerability and Resilience in women with arthritis: Test of a Two-Factor Model. Journal of Consulting and Clinical Psychology, 76(5), 799-810

Stanton, A. L., Reverson, T. A., \& Tennen, H. (2007). Health Psychology: Psychological Adjustment to Chronic Disease. The Annual Review of Psychology, 58, 565-92.

Strand, E. B., Zautra, A. J., Thoresen, M., Odegard, S., Uhlig, T., \& Finset, A. (2006). Positive affect as a factor of resilience in the pain-negative affect relationship in patients with rheumatoid arthritis. Journal Psychosomatic Research, 60(5), 477-484.

Vinaccia, S. y Quiceno, J. M. (2011). Resiliencia y calidad de vida relacionada con la salud en pacientes con insuficiencia renal. Revista Argentina de Clínica Psicológica, 20, 201-211.

Woollet, N.; Cluver, L.; Hatcher, A.; \& Brahmbhatt, H. (2016). To be HIV positive is not the end of the world: Resilience among perinatally infected HIV positive adolescents in Johannesburg. Children and Youth Services Review, 70, 269-275

Yi, J. P., Vitaliano, P. P., Smith, R. E., Yi, J. C., \& Weinger, K. (2008). The role of resilience on psychological adjustment and physical health in patients with diabetes. British Journal of Health Psychology, 13, 311-325

Zautra, A. J., Johnson, L.M., \& Davis, M. C. (2005). Positive Affect as a Source of Resilience for Women in Chronic Pain. Journal of Consulting and Clinical Psychology, 73(2), 212-220.

Zautra, A., Hall, J. \& Murray, K. (2008). Resilience: a new integrative approach to health and mental health research. 
Health Psychology Review, 2(1), 41-64.

Doi: 10.1080/17437190802298568

Zavaleta A. (2012). Resiliencia y programas

preventivos del consumo de drogas.

Edit. Interamericana: La Habana, Cuba.
Todos los trabajos publicados en

Revista Salud \& Sociedad (ISSNe:0718-7475)

están sujetos a una licencia Creative Commons

Reconocimiento 4.0 Internacional

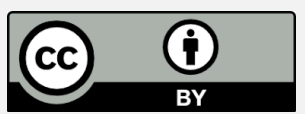

\section{RESUMO}

Objetivo: Avaliar os níveis de resiliência em mexicanos diagnosticados com HIV e comparar os resultados com base em características sociodemográficas e clínicas. Método: Estudo quantitativo, não experimental, de corte transversal, com escopo descritivo e comparativo, com 78 mexicanos com diagnóstico de HIV. Utilizou-se a Escala de Resiliência Mexicana (RESI-M) composta por 43 itens e seis dimensões: Força e Confiança em Si, Competência Social, Suporte à Família, Suporte Social e Estrutura Pessoal; 0 aplicativo foi eletronicamente através da plataforma Google Documents. Resultados: 67,9\% dos avaliados apresentaram alta resiliência, 28,2\% moderado e apenas $3,8 \%$ baixo. Não foram encontradas diferenças significativas em nenhuma das variáveis sociodemográficas a serem comparadas: sexo $(\mathrm{t}=.657, \mathrm{p}=.513)$, faixa etária $(\mathrm{F}=.553, \mathrm{p}=.648)$, escolaridade $(\mathrm{F}=.657 ; \mathrm{p}=.082)$, e antiguidade com a doença ( $F=0,657 ; p=0,802)$. Conclusões: As pessoas com HIV avaliadas para este estudo possuem alta capacidade de resiliência. Apesar de não encontrar diferenças significativas, os homens com mais de 35 anos, com formação universitária e mais tempo com 0 diagnóstico positivo, acabam por ser mais resistentes do que as mulheres.

PALAVRAS-CHAVE: Resiliência, HIV, Psicologia da Saúde.

\section{RESUMEN}

Objetivo: Evaluar los niveles de resiliencia en mexicanos diagnosticados con VIH y comparar los resultados a partir de las características sociodemográficas y clínicas. Método: Estudio de corte cuantitativo, no experimental, de carácter transver sal, con un alcance descriptivo y comparativo, con 78 mexicanos diagnosticados con VIH. Se utilizó la Escala de Resiliencia Mexicana (RESI-M) conformada por 43 items y seis dimensiones: Fortaleza y Confianza en sí mismo, Competencia Social, Apoyo Familiar, Apoyo Social, y Estructura Personal; la aplicación fue de manera electrónica a través de la plataforma Google Documents. Resultados: El 67.9\% de los evaluados arrojaron alta resiliencia, el $28.2 \%$ moderada y solo un $3.8 \%$ niveles bajos. No se encontraron diferencias significativas en ninguna de las variables sociodemográficas a comparar: sexo $(t=.657 ; p=.513)$, rango de edad $(F=.553 ; p=.648)$, escolaridad $(F=.657 ; p=.082)$, y antigijedad con la enfermedad ( $F=.657 ; p=.802$ ). Conclusiones: Las per sonas con VIH evaluadas para este estudio cuentan con una alta capacidad de resiliencia. A pesar de no encontrar diferencias significativas, los hombres con una edad mayor a los 35 años, con escolaridad universitaria y mayor tiempo con el diagnostico positivo, resultan ser más resilientes que las mujeres.

PALABRAS CLAVE: Resiliencia, VIH, Psicología de la salud. 